\title{
Synovial Sarcoma Arising in the Chest Wall Following Ten Years Unexplained Chest Pain
}

\author{
Hideyasu Kudo ${ }^{1}$, Tsutomu Inaoka ${ }^{1 *}$, Wataru Tokuyama ${ }^{2}$, Nobuyuki Hiruta ${ }^{2}$, \\ Akiteru Hayashi ${ }^{3}$, Hitoshi Terada ${ }^{1}$ \\ ${ }^{1}$ Department of Radiology, Toho University Sakura Medical Center, Sakura, Japan \\ ${ }^{2}$ Department of Surgical Pathology, Toho University Sakura Medical Center, Sakura, Japan \\ ${ }^{3}$ Department of Plastic and Reconstructive Surgery, Toho University Sakura Medical Center, Sakura, Japan \\ Email: *tsutomu.inaoka@med.toho-u.ac.jp
}

Received June 2, 2012; revised July 1, 2012; accepted July 11, 2012

\begin{abstract}
Synovial sarcomas are the fourth most common malignant soft-tissue tumors, and typically develop in para-articular locations of the extremities. Other less common sites include the head and neck, abdominal wall, intra-abdominal cavity, and mediastinum. However, the occurrence of these tumors in the chest wall is rare. In this article, we report the interesting case of a 31-year-old male with monophasic synovial sarcoma arising in the left anterior chest wall, who had sustained an unexplained, intermittent sharp pain in the area over a period of ten years before the development of the tumor. We discuss his clinical and radiological findings and include a brief review of the literature.
\end{abstract}

Keywords: Synovial Sarcoma; Pain; Chest; CT; MRI

\section{Introduction}

Synovial sarcomas are the fourth most common type of soft-tissue sarcomas, constituting approximately 5\% $10 \%$ of all soft-tissue sarcomas [1-6]. Synovial sarcomas occur most frequently in adolescents and young adults, with the majority of patients presenting at 15 - 40 years of age [1,3-5,7]. Despite its nomenclature, the tumors do not arise from the synovium [1-6]. Although the etiology of these tumors remains unknown, it is currently speculated that they may derive from mutated, pluripotent mesenchymal cells, which are present in various locations [2,3]. Histologic subtypes include monophasic, biphasic, and poorly differentiated, but the cytogenetic aberration of $\mathrm{t}(\mathrm{X} ; 18)$ translocation is highly specific for synovial sarcomas $[1,2,4-6]$. The vast majority $(80 \%$ $95 \%$ ) of synovial sarcomas occur in the deep soft tissue of the extremities, with two-thirds being located in the lower limbs [1-4,6]. Other less common sites include the head and neck, abdominal wall, intra-abdominal cavity, and mediastinum [2-4]; however, these tumors rarely arise in the chest wall [2-4,6].

Synovial sarcomas may be generally recognized as intermediate- to high-grade soft-tissue sarcomas characterized by local invasiveness and a tendency to metastasize early, especially to the lungs and lymph nodes. However, some synovial sarcomas may be small in size and slow

*Corresponding author. growing, which may lead to an initial false diagnosis of benign indolent lesion $[1,3]$. The clinical presentation of synovial sarcoma is typically a palpable soft-tissue mass or swelling $[1,3,7,8]$. If the lesions are located in deeper sites, symptoms from neurovascular or bony compression may occur [1,3]. In the English literature, however, pain preceding a palpable soft-tissue mass or swelling has been briefly described as a unique clinical feature of synovial sarcomas $[1,3,7,8]$. Approximately $30 \%$ of patients with synovial sarcoma reportedly show this unusual presentation $[7,8]$. In the present article, we report the interesting case of a 31-year-old male with monophasic synovial sarcoma arising in the left anterior chest wall. The patient had sustained an unexplained, intermittent sharp pain in the area over a period of ten years before the development of the tumor. We discuss his clinical and radiological findings and include a brief review of the literature.

\section{Case Report}

A 31-year-old male presented at our hospital with a palpable soft-tissue mass lesion and sharp pain in the left anterior chest wall. There was no history of trauma or preexisting disease. He explained that he had been having chest pains since he was 21 years old and had previously come to our hospital when he was 27 years old because the chest pain had worsened. According to the 
medical records from that visit, he had described the chest pain as localized at the left anterior chest wall, and had stated that it was intermittent and aggravated by pressure. On physical examination, there was only a localized pain with pressure at the left anterior chest wall with no soft-tissue swelling. Chest radiography and echocardiography were negative. Therefore, a diagnosis of peripheral neuropathy was made and the patient was treated with medication. After that, however, the chest pain had again worsened. During the several months prior to his most recent visit to our hospital, the patient noticed a growing palpable soft-tissue mass lesion with sharp pain in the same region. The pain was intermittent but significantly aggravated by pressure. On physical examination, there was a subcutaneous palpable mass lesion located just below the left nipple. No skin pigmentation was found. Chest computed tomography (CT) showed a relatively well-defined, elliptical-shaped, homogenous soft-tissue mass lesion measuring $3.5 \mathrm{~cm}$ in the subcutaneous area of the left anterior chest wall between the $5^{\text {th }}$ and $6^{\text {th }}$ ribs. There was no evidence of calcification. The left $5^{\text {th }}$ and $6^{\text {th }}$ ribs appeared to be normal (Figure 1). On magnetic resonance imaging (MRI), the mass lesion showed homogenous high signal intensity on T2-weighted imaging (T2WI), intermediate signal intensity on T1WI, and homogenously strong enhancement on contrast-enhanced T1WI with fat suppression. Decreased diffusion is seen on diffusion-weighted image (DWI) (Figure 2). Therefore, our impression based on imaging was that the patient had a benign or malignant neurogenic tumor. Aspiration biopsy was initially performed, but no diagnosis was established. The pain had been getting considerably worse, and surgical tumor resection was performed. The surgical records showed that the mass lesion was located anterior to the left $5^{\text {th }}$ and $6^{\text {th }}$ ribs and the intercostal ligaments. No obvious invasion of the ribs or intercostal nerves was identified. Based on the pathological specimens, the tumor was identified as a malignant mesenchymal spindle-cell tumor (Figure 3).

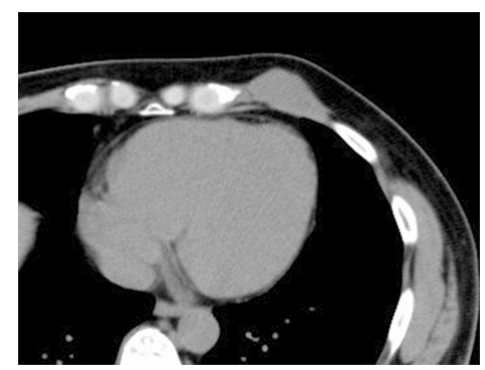

Figure 1. Chest CT. CT showed a relatively well-defined, elliptical-shaped, homogenous soft-tissue mass lesion measuring $3.5 \mathrm{~cm}$ in the subcutaneous area of the left anterior chest wall between the $5^{\text {th }}$ and $6^{\text {th }}$ ribs. There was no evidence of calcification. The $5^{\text {th }}$ and $6^{\text {th }}$ ribs appeared to be normal.

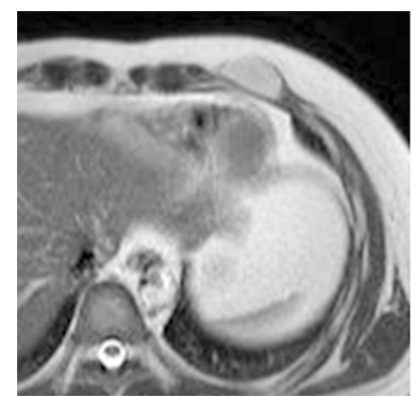

(a)

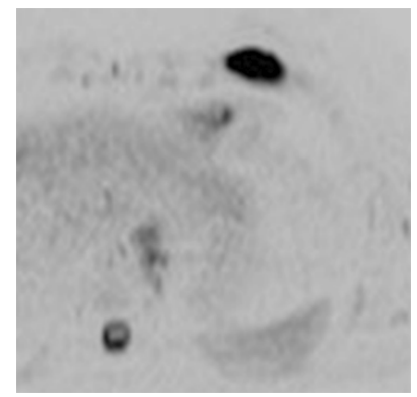

(c)

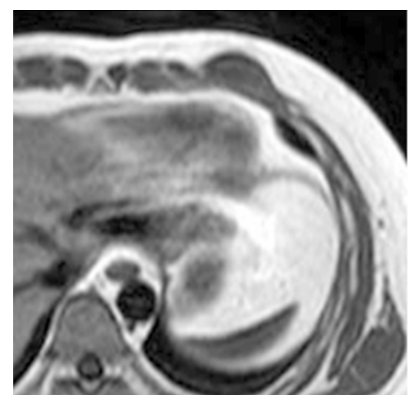

(b)

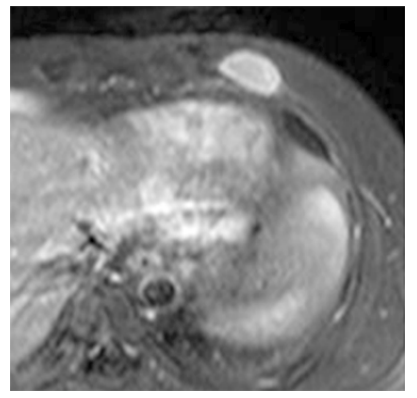

(d)

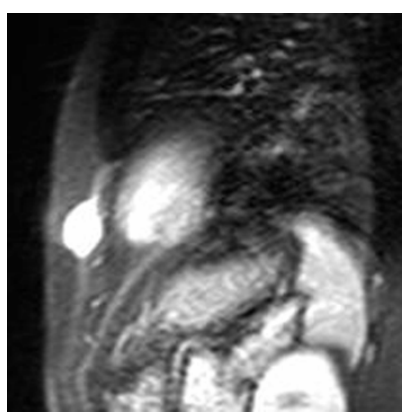

(e)

Figure 2. MRI (a) Axial T2WI; (b) Axial T1WI; (c) Axial DWI (reversed image); and (d) Axial and (e) Sagittal contrast-enhanced T1WI with fat suppression. The mass lesion showed homogenous bright signal intensity on T2WI, intermediate signal intensity on T1WI, and homogenously strong enhancement on contrast-enhanced T1WI with fat suppression. Decreased diffusion is seen on DWI. The mass lesion is located anterior to the $5^{\text {th }}$ and $6^{\text {th }}$ ribs and the intercostals ligament, but there is no evidence of obvious invasion to the ribs. There is no evidence of associated tumorous edema.

Immunohistochemical staining was positive for cytokeratin 7, Bcl-2, MIC-II (CD99), and NCAM (CD56). The tumor was negative for epithelial membrane antigen (EMA), S100 protein, and alpha-smooth muscle actin (SMA). Although EMA is commonly positive in synovial sarcomas, based on the microscopic findings and other immunohistochemical staining, a diagnosis of primary synovial sarcoma (monophasic subtype) was established. The RNA from the tumor was subjected to a polymerase chain reaction amplification and SS18-SSX2 fusion gene transcripts were confirmed. The patient then underwent 


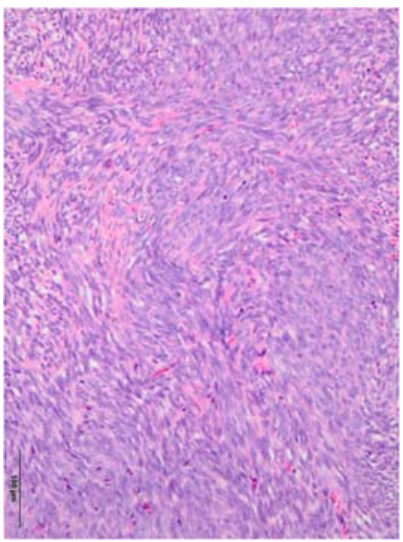

(a)

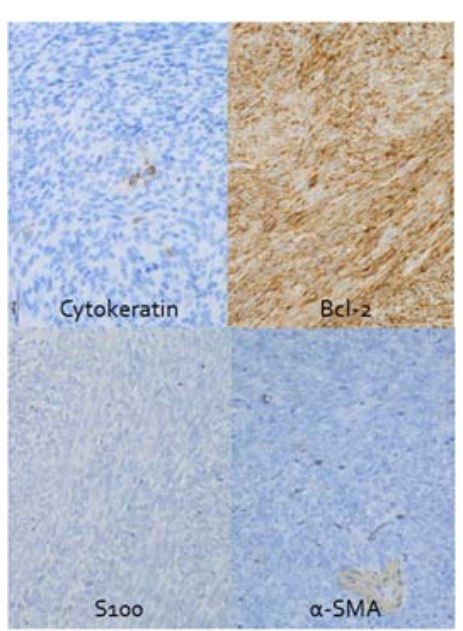

(c)

Figure 3. (a) $\mathrm{H}$ and $\mathrm{E}, \times 100$, (b) $\mathrm{H}$ and $\mathrm{E}, \times 200$, (c) Immunochemical stain. Photomicrograph of a pathological specimen shows a malignant spindle-cell component with pleomorphic nuclei. Immunochemical stains show focal positive for cytokeratin 7, positive for Bcl-2, and negative for S100 protein and alpha-SMA.

surgical wide resection. Adjuvant chemotherapy and radiation therapy were attempted, but the patient rejected them. There was no evidence of local recurrence or metastasis at follow-up after 8 months, but careful observation is required.

\section{Discussion}

There are three main histologic subtypes of synovial sarcomas: monophasic, biphasic, and poorly differentiated $[1,3,4]$. Monophasic subtypes represent 50\% - 60\% of all such lesions, and within this subtype the mesenchymal spindle cell component predominates [1,4]. The monophasic and biphasic subtypes are both usually intermediate grade, but both can be high grade [1]. The poorly differentiated subtype is usually high grade. The hallmarks for synovial sarcomas are the $\mathrm{t}(\mathrm{X} ; 18)$ translocation and SS18-SSX gene fusion products. This genetic aberration has been identified in greater than $90 \%$ of synovial sarcomas regardless of subtypes and is highly specific $[1,2,4-6]$. Several variations of the $t(X ; 18)$ translocation leading to two different gene fusions have been described and are known to be associated with the vast majority of synovial sarcomas [1,4-6]. The SS18SSX1 and SS18-SSX2 types account for 67\% and 33\% of gene fusions, respectively [1,4]. Monophasic tumors are either SSX1 or SSX2, and biphasic tumors are generally SSX1 [1,4].

Patients with synovial sarcomas commonly present with a palpable soft-tissue mass or swelling [1,3,7]. Since the mass lesions sometimes grow slowly, they may give the false impression of a benign process [1,3,7]. This leads to a long duration of symptoms before the correct diagnosis is made; the average is 2 - 4 years [1,7]. Pain and point tenderness at the site of the mass lesion are more frequent than thought. Interestingly, approximately $30 \%$ of synovial sarcoma patients present with pain but no palpable mass at the initial consultation. This phenomenon is much more common in synovial sarcomas than in the case of other soft-tissue sarcomas that typically manifest as painless masses [7,8].

In general, clinical symptoms including pain from soft-tissue sarcomas are due to expansion, stretching, and destruction of surrounding tissues as well as hemorrhage and necrosis within the tumors. In the setting of primary or secondary involvement of the adjacent nerves, pain and sensory and motor dysfunction distal to the lesion may occur [7]. In synovial sarcomas, however, point pain is occasionally present before the development of tumors $[7,8]$. Some investigators have speculated that the pain might be intrinsically related to the biological behavior of synovial sarcomas, including the release of cytokines, prostaglandins, and other inflammatory mediators [7]. In the present case, pathological findings such as necrosis or hemorrhage were not prominent within the lesion.

Synovial sarcomas arising within major or minor nerves have been reported in 22 cases [5]. These cases frequently presented with pain and sensory and/or motor disturbance. Interestingly, most synovial sarcomas of the nerves are of the monophasic type (90\%) and show the SS18-SSX2 fusion gene (60\%), suggesting that synovial sarcomas of the nerves have some unique features [5]. Imaging findings of synovial sarcomas of nerve are reportedly similar to those of benign or malignant neurogenic tumors $[3,5,9]$. In the present case, operative and pathological findings showed no obvious involvement of nerves or arteries such as the intercostal nerves or arteries. Therefore, we cannot clearly explain the reason for the pain before the development of the tumor. However, neurovascular encasement from synovial sarcomas is not unusual and has been reported in $17 \%$ - 24\% of cases. Thus, synovial sarcomas may affect a nerve or neurovas- 
cular bundle primarily or secondarily compared to other soft-tissue tumors arising in the deeper sites [1]. Further studies are needed to elucidate the mechanism of pain before the development of synovial sarcomas.

Imaging findings of synovial sarcomas are commonly nonspecific. Radiographs show round to oval juxtaarticular soft-tissue masses. Calcification is identified in up to $30 \%$ of synovial sarcomas upon radiography. Involvement of the underlying bone is not uncommon, and extrinsic erosion of the bone or a periosteal reaction has been reported in $11 \%$ - $20 \%$ of synovial sarcomas. CT shows a deep-seated, well-circumscribed, heterogeneously enhanced lesion [1,3]. On MRI, T1WI shows a predominantly heterogenous multilobulated soft-tissue mass with signal intensity similar to or slightly higher than that of muscle. T2WI shows prominent heterogeneity with predominant high signal intensity. Hemorrhage or cystic changes are frequently found $[1,3,6]$. Contrastenhanced T1WI shows heterogenous, prominent enhancement. Smaller lesions (less than $5 \mathrm{~cm}$ ) at presentation may have a predominantly homogenous appearance on all MR images, regardless of pulse sequence, a finding that simulates a less aggressive process $[1,3,5]$. There is no notable difference between the MRI characteristics of the monophasic and biphasic subtypes [3]. PET/CT sometimes demonstrates increased uptake of FDG in the lesion [2].

The treatment of choice for synovial sarcomas is wide local excision. The role of adjuvant therapy in the treatment of synovial sarcoma remains controversial. Chemotherapy has been used to treat metastatic or residual disease. Studies have shown a limited survival benefit for high-risk patients following adjuvant chemotherapy. Radiation therapy plays an important role in the treatment of marginally resected tumors [1,2,6]. Patients with synovial sarcomas have a 5-year survival rate ranging from $36 \%$ to $76 \%[1,2,4,7]$. However, the poorly differentiated subtype is associated with a worse prognosis, with a 5 -year survival rate of only $20 \%-30 \%$. There is no distinct difference in outcome between the monophasic and biphasic subtypes, although this remains controversial. More recently, SYT-SSX2 gene fusion has been associated with a better prognosis and an $89 \%$ metastasis-free survival [1]. As in the present case, awareness of the unusual clinical presentation of pain preceding tumor development may lead to improved survival as a result of earlier tumor detection.

In conclusions, we have presented here the interesting case of a 31-year-old male with monophasic synovial sarcoma arising in the left anterior chest wall, and who had suffered an unexplained, intermittent sharp chest pain over a period of ten years before the development of the tumor. The imaging findings were similar to those of peripheral neurogenic tumors. This unique phenomenon of pain before the development of the tumor is reportedly much more common in the cases of synovial sarcomas in contrast to in those of other malignant soft-tissue tumors. If a soft tissue lesion would present this unique clinical symptom, synovial sarcomas should be included in the differential diagnosis.

\section{REFERENCES}

[1] M. D. Murphey, M. S. Gibson, B. T. Jennings, et al., "Imaging of Synovial Sarcoma with Radiologic-Pathologic Correlation,” Radiographics, Vol. 26, 2006, pp. 1543-1565. doi:10.1148/rg.265065084

[2] A. A. Frazier, T. J. Franks, R. D. Pugatch and J. R. Galvin, "Pleuropulmonary Synovial Sarcoma," Radiographics, Vol. 26, 2006, pp. 923-940. doi:10.1148/rg.263055211

[3] P. J. O’Sullivan, A. C. Harris and P. L. Munk, "Radiological Features of Synovial Cell Sarcoma,” British Journal of Radiology, Vol. 81, No. 964, 2008, pp. 346-356. doi:10.1259/bjr/28335824

[4] D. Kawano, I. Yoshino, F. Shoji, et al., "Synovial Sarcoma of the Chest Wall," General Thoracic Cardiovascular Surgery, Vol. 58, No. 2, 2010, pp. 95-97. doi:10.1007/s11748-009-0480-7

[5] B. W. Scheithauer, K. K. Amrami, A. L. Folpe, et al., "Synovial Sarcoma of Nerve," Human Pathology, Vol. 42, No. 4, 2011, pp. 568-577. doi:10.1016/j.humpath.2010.08.019

[6] L. T. Bui-Mansfield, K. J. Kaplan and J. Boardman, "Synovial Sarcoma of the Chest Wall," American Journal of Roentgenology, Vol. 179, 2002, p. 880.

[7] M. V. C. De Silva, A. Barrett and R. Reid, "Premonitory Pain Preceeding Swelling: A Distinctive Clinical Presentation of Synovial Sarcoma Which May Prompt Early Detection,” Sarcoma, Vol. 7, No. 3-4, 2003, pp. 131-135. doi:10.1080/13577140310001644788

[8] N. L. Cadman, E. H. Soule and P. J. Kelly, "Synovial Sarcoma: An Analysis of 134 Tumours," Cancer, Vol. 18, No. 5, 1965, pp. 613-627.

doi:10.1002/1097-0142(196505)18:5<613::AID-CNCR28 20180510>3.0.CO;2-V

[9] K. R. Shahid, K. K. Amrami and R. J. Spinner, "Primary Monophasic Synovial Sarcoma Presenting as a Benign Neurogenic Timor: Case Review and Review of the Literature," Journal of Surgical Orthopaedic Advances, Vol. 19, No. 2, 2010, pp. 129-133. 